\title{
Correction to: Early detection monitoring for non- indigenous fishes; comparison of survey approaches during two species introductions in a Great Lakes port
}

\author{
Greg S. Peterson (D) Joel C. Hoffman • Anett S. Trebitz • Chelsea I. Hatzenbuhler • \\ Jared T. Myers $\cdot$ Jason E. Ross $\cdot$ Sara L. Okum $\cdot$ Erik M. Pilgrim
}

Published online: 13 December 2021

This is a U.S. government work and not under copyright protection in the U.S.; foreign copyright protection may apply 2021

Correction to: Biol Invasions https://doi.org/10. 1007/s10530-021-02655-9

In the original publication of the article, the Table 4 was published with misalignment and the correct alignment of Table 4 is given in this correction.

The original article has been corrected.

E. M. Pilgrim

Center for Environmental Measurement and Modeling, Watershed and Ecosystem Characterization Division, US Environmental Protection Agency, 26 Martin Luther King Dr, Cincinnati, OH 45268, USA

\section{Duluth, MN 55804, USA}

Environmental Protection Agency, 6201 Congdon Blvd,

e-mail: Peterson.Greg@epa.gov

\section{I. Hatzenbuhler}

SpecPro Professional Services, Contractor To US

Environmental Protection Agency, 6201 Congdon Blvd,

Duluth, MN 55804, USA

J. T. Myers - J. E. Ross

US Fish and Wildlife Service, Ashland Fish and Wildlife Conservation Office, 2800 Lakeshore Drive E, Ashland, WI 54806, USA

\section{S. L. Okum - E. M. Pilgrim}

Pegasus Professional Services, Contractor To US

Environmental Protection Agency, 26 Martin Luther King

Dr, Cincinnati, OH 45268, USA 
Table 4 Number of White bass and Gizzard shad detection sites by year and season, for different early detection monitoring survey approaches

\begin{tabular}{llllll}
\hline Species; survey approach & Survey season & \multicolumn{2}{l}{ Survey year } & & \\
\cline { 3 - 6 } & & 2015 & 2016 & 2017 & 2018 \\
\hline White bass; Ichthyoplankton & Summer & 10 & 7 & 5 & NS \\
White bass; Adult-Juvenile & Spring & NS & 0 & 0 & $1(1)$ \\
& Summer & $2(2)$ & $1(1)$ & $8(35)$ & $4(6)$ \\
& Fall & NS & $4(7)$ & $6(9)$ & 0 \\
White bass; eDNA & Summer & NS & 1 & NS & 1 \\
Gizzard shad; Ichthyoplankton & Summer & NS & 0 & NS & 1 \\
Gizzard shad; Adult-Juvenile & Spring & NS & 0 & 0 & NS \\
& Summer & 0 & 0 & 0 & 0 \\
Gizzard shad; eDNA & Fall & NS & 0 & 0 & 0 \\
& Summer & NS & 0 & NS & 1 \\
& Fall & NS & 3 & NS & 3 \\
\hline
\end{tabular}

Publisher's Note Springer Nature remains neutral with regard to jurisdictional claims in published maps and institutional affiliations. 